\title{
Abscess Fluid
}

National Cancer Institute

\section{Source}

National Cancer Institute. Abscess Fluid. NCI Thesaurus. Code C150890.

The fluid that is abnormally collected in localized areas of damaged tissue within the body, usually composed of living and dead white blood cells, bacteria, and dead tissue. 